\title{
A CONTRIBUTION TO THE THEORY OF FUNDAMENTAL TRANSFORMATIONS OF SURFACES*
}

\author{
BY \\ M. M. SLOTNICK

\section{INTRODUCTION}

Two surfaces are said to be related to one another by a fundamental transformation, that is, by a transformation $F$, if the developables of the congruence of lines joining corresponding points on the surfaces cut the surfaces in conjugate nets of curves. It is assumed that neither of these nets is a focal net of the congruence. The nets on the surfaces are also said to correspond by the transformation $F$.

Although many well known transformations of surfaces are special types of transformations $F$, the general case was treated in detail but recently, by Eisenhart $\dagger$ and Jonas. $\ddagger$ In a recent paper Graustein $\S$ introduced into the study of these transformations a projective invariant which was the generalization of the invariant of a parallel map.\| Certain important theorems concerning this invariant were obtained whose nature indicates that transformations $F$ can be investigated to advantage by means of it. We call this invariant the invariant $C$.

When studied in terms of tangential coördinates, transformations $F$ present a complete duality among the elements involved. In this way, a second invariant, the invariant $H$, is obtained which is dual to the invariant $C$. The invariant $C$ is equal to the cross ratio in which a pair of corresponding points of the surfaces in the relation $F$ is divided by the focal points of the line joining them. Dually, the invariant $B$ is equal to the cross ratio in which a pair of corresponding tangent planes to the two surfaces is divided by the focal planes through their line of intersection.

* Presented to the Society, October 29, 1927; received by the editors June 11, 1927.

$\dagger$ Cf. Eisenhart's treatise, Transformations of Surfaces, Princeton, 1923, which deals primarily with these transformations. We shall follow the notation employed in this book, and shall refer to it as Eisenhart, T. S.

¥ Jonas, Sitzungsberichte, Berliner Mathematische Gesellschaft, vol. 14 (1915), pp. 103 ff.

$\$$ W. C. Graustein, $A n$ invariant of a general transformation of surfaces, Bulletin of the American Mathematical Society, vol. 32 (1926), p. 357 ff.

II W. C. Graustein, Parallel maps of surfaces, these Transactions, vol. 23 (1922), pp. 298-332. 
It is the purpose of this paper to make a study of transformations $F$ based upon these invariants. In fact, the invariants $C$ and $B$ form a tool by means of which many theorems are found which do not easily lend themselves to proof by the classical methods. Fundamental existence questions which arise concerning the conditions on the invariants $C$ and $B$ and on the nets in the transformation $F$ are readily answered. The relations between the invariants $C$ and $B$ and the surfaces in the transformation also yield interesting consequences.

The invariant $C$ is introduced in Part I, which also contains a fundamental theorem for transformations $F$ of a given net having a given invariant $C$. The analogous work for the invariant $B$ is done in Part II. The invariants $C$ and $B$ of a transformation $F$ which is the product of two such transformations are also discussed in these two parts.

Transformations $F$ and nets of special type are discussed in Part III. The last part, Part IV, is devoted to the application of some of the results obtained to transformations of Ribaucour.

\section{The invariant $C$ of a transformation $F$}

1. Fundamental equations. A congruence of lines $G$ and a net $N$ are said to be conjugate to one another if the curves of $N$, which is assumed not a focal net of $G$, lie on the developables of $G$. Two nets $N$ and $N_{1}$ are then related to one another by a transformation $F$ if the congruence $G$ of lines joining corresponding points of these nets is conjugate to both nets. The congruence $G$ is known as the conjugate congruence of the transformation $F$.

Consider a surface $S: x=x(u, v)^{*}$ on which the parametric curves form a net $N$, which has for its point equation

$$
\frac{\partial^{2} \theta}{\partial u \partial v}=\frac{\partial \log a}{\partial v} \frac{\partial \theta}{\partial u}+\frac{\partial \log b}{\partial u} \frac{\partial \theta}{\partial v} \cdot \dagger
$$

To obtain an $F$ transform of $N$ we have first to find a congruence $G$ conjugate to $N$, and then a net $N_{1}$ conjugate to $G$. A net $N^{\prime}$, parallel to $N$, and traced by the point $x^{\prime}$, where

$$
\frac{\partial x^{\prime}}{\partial u}=l \frac{\partial x}{\partial u}, \quad \frac{\partial x^{\prime}}{\partial v}=l \frac{\partial x}{\partial v},
$$

determines $G$ in that the point coördinates of $N^{\prime}$ serve as direction parameters

* I.e., $x^{i}=x^{i}(u, v), i=1,2, \cdots, n$.

$\dagger$ Eisenhart, T. S., \& 2. 
for the lines of $G$. A solution $\theta$ of the point equation (1.1) of $N$ will determine a net $N_{1}$, conjugate to $G$, whose point coördinates are

$$
x_{1}=x-\frac{\theta}{\theta^{\prime}} x^{\prime} .
$$

Here $\theta^{\prime}$ is a solution of the point equation of $N^{\prime}$ corresponding to $\theta$; i.e., it satisfies the equations

$$
\frac{\partial \theta^{\prime}}{\partial u}=h \frac{\partial \theta}{\partial u}, \quad \frac{\partial \theta^{\prime}}{\partial v}=l \frac{\partial \theta}{\partial v} .
$$

The net $N_{1}$ is said to be an $F$ transform of $N$ by means of the solution $\theta$ of its point equation and along the congruence $G$.

The lines of intersection of corresponding tangent planes to the surfaces of $N$ and $N_{1}$ also generate a congruence called the harmonic congruence of the transformation. For a line $L$ of this congruence the focal points $F_{1}, F_{2}$ have the coördinates*

$$
F_{1}: x-\frac{\theta}{\frac{\partial \theta}{\partial u}} \frac{\partial x}{\partial u}, \quad F_{2}: x-\frac{\theta}{\frac{\partial \theta}{\partial v}} \frac{\partial x}{\partial v} ;
$$

and hence are the intersections of $L$ with the focal planes of the corresponding line of $G$.

2. The invariant $C . \dagger$ A transformation $F$ of the net $N$ into the net $N_{1}$ establishes a projective correspondence between the pencils of the tangent lines to the surfaces of these nets at corresponding points $x$ and $x_{1}$. These pencils of tangent lines meet the line of intersection $L$ of their planes (the tangent planes to the surfaces of $N$ and $N_{1}$ at $x$ and $x_{1}$, respectively) in projective ranges of points. In this projectivity the fixed points are the focal points $F_{1}$ and $F_{2}$. If $D$ and $D_{1}$ are a pair of corresponding points of the two ranges on $L$, the invariant of the projectivity is

$$
C=\left(D D_{1}, F_{1} F_{2}\right) .
$$

The function $C$ is a projective invariant of the transformation $F$ which we shall call the conjugate invariant, or, briefly, the invariant $C$.

The invariant $C$ has another geometric significance. $\ddagger$ It is the cross ratio

* Eisenhart, T. S., \& 17.

$\dagger$ W. C. Graustein, $A n$ invariant of a general transformation of surfaces, 85.

$\ddagger$ Ibid., 83. 
in which the points $x$ and $x_{1}$ of the nets $N$ and $N_{1}$ are divided by the focal points $z$ and $y$ of the line of $G$; i.e.,

$$
C=\left(x x_{1}, z y\right) \text {. }
$$

For the transformation $F$ discussed in $\$ 1$, the invariant $C$ is found to be

$$
C=\frac{t}{s}, *
$$

where

$$
t=h \theta-\theta^{\prime}, \quad s=l \theta-\theta^{\prime} .
$$

Two nets are said to be radial transforms $†$ of one another when the lines joining corresponding points are concurrent. We agree to admit radial transformations into the category of transformations $F$, and point out that $C=1$ is characteristic of them.

Finally, we note that the invariant $C$ of the inverse of a transformation $F$ is equal to the reciprocal of that of the original transformation.

3. Fundamental theorem. Equations (2.3), (2.4), (1.1), and (1.2), combined with the condition of compatibility of the equations (1.2), yield the relations

$$
\begin{aligned}
& \frac{\partial \log t}{\partial v}=\left(1-\frac{1}{C}\right) \frac{\partial}{\partial v} \log \frac{\theta}{a} \\
& \frac{\partial \log s}{\partial u}=(1-C) \frac{\partial}{\partial u} \log \frac{\theta}{b} .
\end{aligned}
$$

We now form the difference between the derivative of the first of these equations with respect to $u$ and that of the second with respect to $v$ and obtain the equation

$$
\frac{\partial^{2} \log C}{\partial u \partial v}+\frac{\partial}{\partial u}\left[\left(\frac{1}{C}-1\right) \frac{\partial}{\partial v} \log \frac{\theta}{a}\right]+\frac{\partial}{\partial v}\left[(1-C) \frac{\partial}{\partial u} \log \frac{\theta}{b}\right]=0
$$

as a condition on the invariant $C$ and the solution $\theta$ of the point equation of the net $N$, for the transformation $F$.

Suppose now that we have a net $N$ with (1.1) as its point equation, of which $\theta$ is a given solution. Given also a function $C(u, v)$ satisfying (3.2). The system of equations (3.1) combined with $s C=t$ is then compatible,

* Ibid., 85.

† Eisenhart, T. S., \& 14. 
and by means of it two functions $t$ and $s$ are defined to within the same multiplicative constant. The function

$$
\phi=\frac{t-s}{\theta}
$$

is defined also to within this same multiplicative constant, and is found to satisfy the adjoint equation of (1.1), namely

$$
\frac{\partial^{2} \phi}{\partial u \partial v}+\frac{\partial \log a}{\partial v} \frac{\partial \phi}{\partial u}+\frac{\partial \log b}{\partial u} \frac{\partial \phi}{\partial v}+\frac{\partial^{2} \log a b}{\partial u \partial v}=0 .
$$

Consequently the relation

$$
h-l=\phi
$$

will determine two functions $h$ and $l$ to within a common additive constant of integration, which serve to define a net $N^{\prime}:\left(x^{\prime}\right)$ parallel to $N$, by means of equations similar to (1.2).* The function $\theta^{\prime}=h \theta-t=l \theta-s$ is then a solution of the point equation of $N^{\prime}$ corresponding to $\theta$.

Thus we have found transformations $F$ of $N$ by means of the given $\theta$ having the given function $C(u, v)$ as their common invariant $C$. The nets $N_{1}$ determined in this manner as $F$ transforms of $N$ are given by the equation

$$
x_{1}=x-\frac{\theta}{\theta^{\prime}+n \theta}\left(x^{\prime}+n x\right), \dagger
$$

in which $n$ is an arbitrary constant.

Fundamental Theorem I. A solution $\theta$ of the point equation (1.1) of a net $N$, and a function $C(u, v)$ which satisfies (3.2) determine $\infty^{1}$ nets $N_{1}$ which are $F$ transforms of $N$ by means of $\theta$ having as their invariant $C$ the given function $C(u, v)$. Any two of the nets $N_{1}$ are radial transforms of one another.

The last part of the theorem can be proved directly from (3.6); but it will be made evident by the corollary of $\S 6$.

4. Conjugate triads. If $N_{1}$ and $N_{2}$ are $F$ transforms of the net $N$ by essentially different solutions, $\theta_{1}$ and $\theta_{2}\left(\theta_{1} \neq c \theta_{2}\right)$, of its point equation (1.1), but along the same conjugate congruence, they are themselves in relation $F$. The transformation $F$ carrying $N$ into $N_{i}(i=1,2)$ we indicate by $F_{i}$, and that carrying $N_{1}$ into $N_{2}$ by $F_{3}$; then

* Eisenhart, T. S., $\$ 4,(18),(19),(20)$ and also the next theorem stated there.

This result is obtained by availing ourselves of a translation of the coördinate axes. 


$$
F_{1} F_{3}=F_{2} \text {. }
$$

Three nets so related to one another will be referred to as a conjugate triad of nets.

From (1.5) it is noted that the transformations $F_{1}$ and $F_{2}$, and therefore also $F_{3}$, have different harmonic congruences, corresponding lines of which are concurrent.

If we indicate the invariant $C$ of $F_{i}$ by $C_{i}(i=1,2,3)$, we have from (2.2)

$$
C_{1}=\left(x x_{1}, z y\right), \quad C_{2}=\left(x x_{2}, z y\right), \quad C_{3}=\left(x_{1} x_{2}, z y\right) .
$$

Hence

$$
C_{1} C_{3}=C_{2} .
$$

5. Harmonic triads. Suppose now that $N_{1}$ and $N_{2}$ are $F$ transforms of $N$ by means of the same solution $\theta$ of its point equation (1.1), but along different conjugate congruences. The nets $N_{1}$ and $N_{2}$ will be, in this case also, $F$ transforms of one another.* Three nets related to one another in this manner will be referred to as a harmonic triad of nets. Using the same symbolism as in the preceding section we may again write

$$
F_{1} F_{8}=F_{2} \text {. }
$$

Any two of the three nets in a harmonic triad are obtained as $F$ transforms of the third by means of the same solution of its point equation. Because of this fact, we see from (1.5) that the three transformations $F$ involved have the same harmonic congruence. It is to be noted also that the transformations $F$ in a harmonic triad of nets have different conjugate congruences, corresponding lines of which are coplanar.

Using the definition of $C$ as embodied in (2.1), we conclude that here, too,

$$
C_{1} C_{3}=C_{2} .
$$

6. Product of two transformations $F$. Suppose that the net $N_{i}$ is transformed into the net $N_{j}$ by the transformation $F_{k}(i, j, k=1,2,3$ cyclically), and let $L_{k}$ be a line of the harmonic congruence of $F_{k}$. Since $L_{k}$ is the intersection of the tangent planes to $N_{i}$ and $N_{i}$, the three lines $L$ must be either concurrent, or all three coincident. If the lines $L$ are concurrent, the triangle formed by the focal points at the intersections of the tangents to the $u$-curves with one another (cf. (1.5)) will be in the relation

* Eisenhart, T. S., \& 20. Eisenhart has applied the term triad to what we call a harmonic triad, and has given no name to our conjugate triad. These terms have been introduced in the light of the duality existing between the two types of triads. 
of Desargues with that formed by the other three focal points. Each of the sides of one of these triangles intersects the corresponding side of the other triangle in a point of one of the three nets. Hence, corresponding points of the three nets are collinear and the three nets form a conjugate triad.

If corresponding lines $L$ are coincident, the three transformations $F$ have the same harmonic congruence; that is, the three nets form a harmonic triad.*

This result combined with those of $\S \S 4,5$, yields

THEOREM II. If the product of two transformations $F$ is a transformation $F$, the three nets in question form either a conjugate or a harmonic triad; and, in either case, the invariant $C$ of the product transformation is equal to the product of those of the two given transformations.

As an immediate consequence, we conclude

COROLlary. If two transformations $F$ of a net by the same solution of its point equation have equal invariants $C$, the two $F$ transforms are radial transforms of one another; and conversely, if two non-radial $F$ transforms of a net $N$ along different conjugate congruences are radial transforms of one another, the two transformations $F$ are by means of the same solution of the point equation of $N$ and have equal invariants $C$.

7. Transformations $F$ in homogeneous point coördinates. The point equation of a net $N$ on a surface $S: x=x(u, v)$ in a space referred to a homogeneous point coördinate system is of the form

$$
\frac{\partial^{2} \theta}{\partial u \partial v}=\frac{\partial \log a}{\partial v} \frac{\partial \theta}{\partial u}+\frac{\partial \log b}{\partial u} \frac{\partial \theta}{\partial v}+c \theta . \dagger
$$

If $\theta$ is a solution of this equation, the point $x_{1}$ defined by

$$
\frac{\partial x_{1}}{\partial u}=t \frac{\partial}{\partial u}\left(\frac{x}{\theta}\right), \quad \frac{\partial x_{1}}{\partial v}=s \frac{\partial}{\partial v}\left(\frac{x}{\theta}\right)
$$

traces a net $N_{1}$ which is an $F$ transform of $N$. The points with the coördinates $\partial x_{1} / \partial u$ and $\partial x_{1} / \partial v$ are the focal points $F_{1}$ and $F_{2}$ (cf. (1.5)) of the line of the

* There is also the case in which the three nets $N$ form both a conjugate and a harmonic triad. Two nets which, with the net $N$ of $\$ 1$, form such a configuration are those along the same congruence conjugate to $N$ and by means of the same solution $\theta$ of the point equation of $N$. However, the two "corresponding" solutions of the point equation of $N^{\prime}$ differ by a constant ( $\theta^{\prime}$ and $\theta^{\prime}+$ constant (cf. (1.4)). Three nets so related may be considered as forming either type of triad.

† Eisenhart, T. S., $\$ 830,37,38$. 
harmonic congruence. The invariant $C$ of the transformation $F$ is found to be

$$
C=\frac{t}{s} .
$$

The condition of compatibility of (7.2) assumes the form (3.1) by virtue of the fact that $\theta$ is a solution of (7.1).

For a radial transformation $C=1$; i.e., $t=s$. In this case, because of (3.1), both $t$ and $s$ are equal to the same constant. Equations (7.2) can then be integrated and

$$
x_{1}=\frac{x}{\theta}+\rho
$$

is obtained as the equation of a radial transformation. Here $\rho$ represents the coördinates of the center of the transformation.

From (3.1) we deduce that the condition on the invariant $C$ in this case is precisely of the same form as (3.2).

Given, conversely, a net $N$ whose point equation is (7.1), a solution $\theta$ of (7.1), and a function $C(u, v)$ satisfying the condition (3.2). Just as in $\$ 3$, we find that a net $N_{1}$ is determined as an $F$ transform of $N$ to within a radial transformation*; and that the invariants $C$ of the transformations $F$ are equal to the given function $C$.

If we replace the coördinates $x$ of $N$ by $\theta x$, where $\theta$ is a solution of (7.1), the point equation assumes a similar form but with $c=0$. $\dagger$ In this event, equations (7.2) become similar in form to those for a parallel map in terms of non-homogeneous coördinates. In fact, as Eisenhart $\ddagger$ points out, the study of transformations $F$ in terms of homogeneous coördinates can be made in this way analytically equivalent to that of parallel maps in terms of nonhomogeneous coördinates. In such a development the invariant $C$ of the transformation $F$ corresponds to the invariant of the parallel map. $\delta$

\section{The Invariant $B$ of a transformation $F$}

8. Nets and transformations $F$ in terms of tangential coordinates. The tangential coördinates of a surface $S$ whose point coördinates are $x=$ $x(u, v) \|$ are the direction cosines of its normal:

\footnotetext{
- Inasmuch as we cannot avail ourselves of a translation as we did in 83 , it cannot be concluded here that there are only $\infty^{1}$ transformations $F$ determined.

$\dagger$ Eisenhart, T. S., \& 37.

$\ddagger$ Ibid., p. 89.

\& W. C. Graustein, Parallel maps of surfaces.

\| From now on we restrict ourselves to three-dimensional space.
} 


$$
\zeta=\frac{1}{D} \frac{\partial x}{\partial u} \times \frac{\partial x}{\partial v}, \quad(\zeta \mid \zeta) \equiv 1, *
$$

and the distance of the tangent plane from the origin:

$$
\omega=(\zeta \mid x) \text {. }
$$

The parametric curves of $S$ will form a conjugate net $N$ if and only if the tangential coördinates $\zeta$ and $\omega$ satisfy an equation of the form

$$
\frac{\partial^{2} \lambda}{\partial u \partial v}=\frac{\partial \log \alpha}{\partial v} \frac{\partial \lambda}{\partial u}+\frac{\partial \log \beta}{\partial u} \frac{\partial \lambda}{\partial v}+\gamma \lambda, \dagger
$$

which is known as the tangential equation of $N$.

Let (8.3) be the tangential equation of the net $N$ of $\S 1$; and let $\zeta, \omega$ be its tangential coördinates. The tangential coördinates of $N_{1}$ will be written $\zeta_{1}, \omega_{1}$. The function $\lambda=\left(\zeta \mid x^{\prime}\right)$ is the fourth tangential coördinate of $N^{\prime}$, and is also a solution of (8.3).

The net $N_{1}^{\prime}$ traced by the point

$$
x_{1}^{\prime}=\frac{x^{\prime}}{\theta^{\prime}}
$$

is a radial transform of $N^{\prime}$ by means of $\theta^{\prime}$, and is parallel to $N_{1} . \ddagger$ Its fourth tangential coördinate is $\lambda_{1}=\left(\zeta_{1} \mid x_{1}^{\prime}\right)$.

The transformation $F$ of $\S 1$ is represented in terms of tangential coördinates by the equations

$$
\begin{array}{lll}
\frac{\partial}{\partial u}\left(\frac{\zeta_{1}}{\lambda_{1}}\right)=\bar{t} \frac{\partial}{\partial u}\left(\frac{\zeta}{\lambda}\right), & \frac{\partial}{\partial v}\left(\frac{\zeta 1}{\lambda_{1}}\right)=\bar{s} \frac{\partial}{\partial v}\left(\frac{\zeta}{\lambda}\right), \\
\frac{\partial}{\partial u}\left(\frac{\omega_{1}}{\lambda_{1}}\right)=\bar{t} \frac{\partial}{\partial u}\left(\frac{\omega}{\lambda}\right), & \frac{\partial}{\partial v}\left(\frac{\omega_{1}}{\lambda_{1}}\right)=\bar{s} \frac{\partial}{\partial v}\left(\frac{\omega}{\lambda}\right) \cdot \S
\end{array}
$$

When we reconcile these equations with those of $\S 1$, we find that

* The inner product of the two triples $x:\left(x^{1}, x^{2}, x^{3}\right), y:\left(y^{1}, y^{2}, y^{3}\right)$ is represented by $(x \mid y)$; and their outer product by $x \times y$. In this way we have $(x \times y \mid z)=(x y z)$, the latter term being the determinant of $x, y$ and $z$. Also

$$
D^{2}=E G-F^{2}=\left(\frac{\partial x}{\partial u} \times \frac{\partial x}{\partial v} \mid \frac{\partial x}{\partial u} \times \frac{\partial x}{\partial v}\right),
$$

where $E, F, G$ are the coefficients of the square of the linear element of $S$.

$\dagger$ Eisenhart, Differential Geometry, \$\& 66, 67.

$\ddagger$ Eisenhart, T. S., $\& 15$.

\& Eisenhart, T. S., \&52, (26). 


$$
\bar{t}=-\frac{e_{1} D_{1} \theta^{\prime 4}}{e D t^{2} s}, \quad \bar{s}=-\frac{g_{1} D_{1} \theta^{\prime 4}}{g D t s^{2}} \cdot *
$$

9. The invariant $H$. At the focal point $F_{1}$ (cf. (1.5)) of the line $L$ of the harmonic congruence, the tangent plane to any ruled surface of that congruence is the focal plane there; and a similar situation exists at the other focal point $F_{2}$. Consider an arbitrary ruled surface of the harmonic congruence. The coördinates of the points of contact, $P$ and $P_{1}$, of the tangent planes to $N$ and $N_{1}$ with this arbitrary ruled surface depend linearly on the value of $d u / d v$ for this ruled surface. Thus, as $d u / d v$ is allowed to vary, there are defined on the line of the harmonic congruence two ranges of points, the one traced by $P$ and the other by $P_{1}$, which are in projective correspondence. The fixed points of this projectivity are $F_{1}$ and $F_{2}$. Accordingly, the invariant of this projectivity

$$
H=\left(P P_{1}, F_{2} F_{1}\right)
$$

is a projective invariant of the transformation $F$ which we call the harmonic invariant, or briefly, the invariant $H$.

Evidently the invariant $H$ of the inverse of a transformation $F$ is the reciprocal of that of the given transformation.

The value of $H$ may be obtained by computing the coördinates of $P$ and $P_{1}$ in terms of point coördinates using the formulas of $\$ 1$. It is found that

$$
H=\frac{e_{1} g}{g_{1} e} \cdot \frac{1}{C} \text {. }
$$

An alternative method of obtaining this result is to use the fact that the cross ratio (9.1) is equal to that in which the tangent planes to $N$ and $N_{1}$ are divided by the focal planes at $F_{1}$ and $F_{2}$. The tangential coördinates of the first two planes mentioned are respectively $\zeta, \omega$ and $\zeta_{1}, \omega_{1}$. Those of the focal planes are proportional to

$$
\frac{\zeta_{1}}{\lambda_{1}}-\bar{t} \frac{\zeta}{\lambda}, \frac{\omega_{1}}{\lambda_{1}}-\bar{t} \frac{\omega}{\lambda} \text { and } \frac{\zeta_{1}}{\lambda_{1}}-\bar{s} \frac{\zeta}{\lambda}, \frac{\omega_{1}}{\lambda_{1}}-\bar{s} \frac{\omega}{\lambda} \cdot \dagger
$$

Hence (9.1) becomes

$$
H=\frac{\bar{t}}{\bar{s}} .
$$

By virtue of (8.6) this result is seen to be equivalent to (9.2).

* Here $e, f, g$ are the coefficients of the second fundamental quadratic form for the surface of the net $N$.

† Eisenhart, T. S. $\$ 52$, (27). 
A somewhat different geometric consideration brings to light another meaning of the invariant $H$. As we leave the point $x$ of $N$ in the direction $d u / d v$, the tangent plane there twists about the conjugate direction. Consequently we can obtain the invariant $H$ just as the invariant $C$ was obtained,* except that each direction is now to be replaced by its conjugate direction. In this way, $F_{1}, F_{2}$ of (2.1) are interchanged; and $D$ and $D_{1}$ are replaced by $P$ and $P_{1}$, where the latter two points are the intersections of the line of the harmonic congruence with those tangent lines at $x$ and $x_{1}$ to the surfaces of $N$ and $N_{1}$ in the directions conjugate to the lines from $x$ and $x_{1}$ to $D$ and $D_{1}$.

10. Perspective transformations. The conditions of integrability of (8.5) can be written in the form

$$
\text { (10.1) } \begin{aligned}
\frac{\partial \log \bar{t}}{\partial v} & =\left(1-\frac{1}{H}\right) \frac{\partial}{\partial v} \log \frac{\lambda}{\alpha}, \\
\frac{\partial \log \bar{s}}{\partial u} & =(1-H) \frac{\partial}{\partial u} \log \frac{\lambda}{\beta},
\end{aligned}
$$

where we have made use of (8.3) and (9.3).

If $H=1, \bar{t}=\bar{s}$ and their common value is seen from equations (10.1) to be constant. Equation (8.5) can be integrated in this case and we obtain

$$
\zeta_{1}=\frac{\zeta+\lambda \rho}{\Lambda}, \quad \omega_{1}=\frac{\omega+\lambda r}{\Lambda} ; \quad \lambda_{1}=\frac{\lambda}{\Lambda},
$$

where

$$
\Lambda=[(\zeta+\lambda \rho) \mid(\zeta+\lambda \rho)]^{1 / 2} .
$$

Hence the lines of intersections of corresponding tangent planes to $N$ and $N_{1}$ all lie in a fixed plane. We refer to a transformation of this type as a perspective transformation.

Inasmuch as a perspective transformation in terms of tangential coördinates is analytically equivalent to a radial transformation in terms of point coördinates, we may say that they are duals of one another.

A simple example of a perspective transformation is the parallel map, in which the plane of perspectivity is the plane at infinity.

11. Fundamental theorem. From equations (10.1) in a manner similar to that of $\$ 3$ we obtain, as the condition on the invariant $B$ of the transformation (8.5),

\footnotetext{
* Cf. \& 2, above.
} 


$$
\frac{\partial^{2} \log H}{\partial u \partial v}+\frac{\partial}{\partial u}\left[\left(\frac{1}{H}-1\right) \frac{\partial}{\partial v} \log \frac{\lambda}{\alpha}\right]+\frac{\partial}{\partial v}\left[(1-B) \frac{\partial}{\partial u} \log \frac{\lambda}{\beta}\right]=0 .
$$

Conversely, given a net $N$ whose tangential equation is (8.3), a solution $\lambda$ of (8.3), and a function $B(u, v)$ satisfying (11.1). Equations (10.1) and $\bar{s} H=\bar{t}$ will then form a compatible system by means of which two functions $\bar{t}$ and $\bar{s}$ are defined to within the same multiplicative constant. The equations

$$
\begin{array}{lll}
\frac{\partial w_{i}}{\partial u}=\bar{t} \frac{\partial}{\partial u}\left(\frac{\zeta^{i}}{\lambda}\right), & \frac{\partial w_{i}}{\partial v}=\bar{s} \frac{\partial}{\partial v}\left(\frac{\zeta^{i}}{\lambda}\right) * & (i=1,2,3), \\
\frac{\partial w_{4}}{\partial u}=\bar{t} \frac{\partial}{\partial u}\left(\frac{\omega}{\lambda}\right), & \frac{\partial w_{4}}{\partial v}=\bar{s} \frac{\partial}{\partial v}\left(\frac{\omega}{\bar{\lambda}}\right) . &
\end{array}
$$

are then compatible; and the functions $w_{k}(k=1,2,3,4)$, so defined, all satisfy the equation

$$
\frac{\partial^{2} w}{\partial u \partial v}=\frac{1}{H} \frac{\partial}{\partial v} \log \frac{\alpha}{\lambda} \frac{\partial w}{\partial u}+H \frac{\partial}{\partial u} \log \frac{\beta}{\lambda} \frac{\partial w}{\partial v} .
$$

The five functions

$$
\begin{aligned}
& \zeta_{1}^{i}=\frac{w_{i}}{(w \mid w)^{1 / 2}}, \quad i=1,2,3, \\
& \omega_{1}=\frac{w_{4}}{(w \mid w)^{1 / 2}}, \quad \lambda_{1}=\frac{1}{(w \mid w)^{1 / 2}} \quad\left[(w \mid w)=w_{1}^{2}+w_{2}^{2}+w_{2}^{2}\right],
\end{aligned}
$$

are solutions of the equation

$$
\begin{aligned}
\frac{\partial^{2} \lambda_{1}}{\partial u \partial v}=\frac{\partial}{\partial v} \log \frac{\alpha \bar{t}}{(w \mid w)^{1 / 2}} \frac{\partial \lambda_{1}}{\partial u} & \\
+ & \frac{\partial}{\partial u} \log \frac{\beta \bar{s}}{(w \mid w)^{1 / 2}} \frac{\partial \lambda_{1}}{\partial v}-\frac{\left(w \times \frac{\partial w}{\partial u} \mid w \times \frac{\partial w}{\partial v}\right)}{(w \mid w)^{2}} \lambda_{1} .
\end{aligned}
$$

From (11.4), $\left(\zeta_{1} \mid \zeta_{1}\right) \equiv 1$. Thus the functions $\zeta_{1}$ and $\omega_{1}$ can be considered as the tangential coördinates of a net $N_{1}$ whose tangential equation will be (11.5). Moreover, since (11.2) assumes the form (8.5) when the functions $w$ are replaced by $\zeta_{1}, \omega_{1}$ and $\lambda_{1}$, as indicated by (11.4), the net $N_{1}$ is an $F$ transform of $N$. The analytic work here is the same as in $\$ 3$, but the inter-

\footnotetext{
$\zeta^{1}, \zeta^{2}, \zeta^{3}$ are the three ordered components of $\zeta$ (cf. (8.1)).
} 
pretation now is that these quadratures determine $\zeta_{1}$ and $\omega_{1}$ only to within a perspective transformation (cf. equations (10.2)).

Fundamental Theorem III. A solution $\lambda$ of the tangential equation (8.3) of a net $N$, and a function $H(u, v)$ which satisfies (11.1) determine nets $N_{1}$ to within a perspective transformation which are $F$ transforms of $N$ by means of $\lambda$; the invariant $H$ of these transformations $F$ is the given function $H(u, v)$.

12. Triads. We have seen that if $N_{1}$ and $N_{2}$ are $F$ transforms of a net $N$ (with (8.3) as its tangential equation) and if $N_{1}$ and $N_{2}$ are themselves in relation $F$, then the nets $N, N_{1}, N_{2}$ form either a harmonic or a conjugate triad.* From the nature of the function $\lambda \dagger$ of (8.5), which we say is the solution of the tangential equation of $N$ used in the transformation $F$, we see that if

(i) the same $\lambda$ is used in (8.5) to obtain $N_{1}$ and $N_{2}$ as $F$ transforms of $N$, the three nets form a conjugate triad; and if

(ii) different solutions $\lambda$ are used, the three nets form a harmonic triad.

We have thus the following dual relations:

If $N_{1}, N_{2}, N_{3}$ are three nets in a conjugate[harmonic] triad, the three transformations $F$ have the same conjugate[harmonic] congruence but different harmonic [conjugate] congruences; any two of the nets are obtained from the third by means of the same solution $\lambda[\theta]$ of its tangential[point] equation, but by different solutions $\theta[\lambda]$ of its point [tangential] equation.

In the same way the invariants $C$ and $H$, and radial and perspective transformations are duals of one another.

The methods used in proving the results embodied in the theorem of $\$ 6$ are applicable to the invariant $H$ also. Consequently we now have the complete

THEOREM IV. If the product of two transformations $F$ is a transformation $F$, the three nets in question form either a conjugate or a harmonic triad. In either case, the invariants $C$ and $H$ of the product transformation are equal respectively to the product of the invariants $C$ and to the product of the invariants $H$ of the given two transformations.

As in $\$ 6$, we also have, dually, the

COROLlaRY. If two transformations $F$ of a net by the same solution of its tangential equation have equal invariants $H$, the two $F$ transforms are per-

* Cf. $\$$ 6, above.

† I.e. $\lambda=\left(\zeta \mid x^{\prime}\right)$, cf. $\&$ 8. Here $\lambda$ is the fourth tangential coördinate of $N^{\prime}$, the net parallel to $N$, whose point coördinates are the direction parameters of the conjugate congruence of the transformation. 
spective transforms of one another; and, conversely, if two non-perspective $F$ transformations with different harmonic congruences of a net $N$ are perspective transforms of one another, the two transformations $F$ are by means of the same solution of the tangential equation of $N$ and have equal invariants $B$.

13. Transformations $F$ in homogeneous tangential coordinates. Analytically, the study of transformations $F$, whether in terms of homogeneous point coördinates, or in terms of homogeneous tangential coördinates, is the same.* The work and fundamental theorem of $\$ 7$ need therefore only to be dually interpreted to obtain the facts for transformations $F$ in terms of homogeneous tangential coördinates.

14. The invariants $C$ and $H$ as products of invariants. The transformation $F$ as set up by Eisenhart $\dagger$ is the product of a parallel transformation $P_{1}$, a radial transformation $R_{2}$, and another parallel transformation $P_{8}$; i.e.,

$$
N \stackrel{P_{1}}{\rightarrow} N^{\prime} \stackrel{R_{2}}{\rightarrow} N_{1}^{\prime} \stackrel{P_{3}}{\rightarrow} N_{1} .
$$

Graustein $\ddagger$ has shown that the invariant $C$ of the product transformation $F$ is equal to the product of the invariants $(C)$ of these factor transformations.

Consider now the case of the invariant $B$. Since $P_{1}$ and $P_{8}$ are parallel, i.e., perspective, transformations, $H_{1}=H_{3}=1$. For $R_{2}, C_{2}=1$, and hence, from (9.2), $H_{2}=e_{1}^{\prime} g^{\prime} /\left(g_{1}^{\prime} e^{\prime}\right)$, the quantities bearing on $N_{1}^{\prime}$ and $N^{\prime}$. From (1.2)

Since

$$
e^{\prime}=h e, \quad g^{\prime}=l g .
$$

$$
\frac{\partial x_{1}^{\prime}}{\partial u}=-\frac{h}{t} \frac{\partial x_{1}}{\partial u}, \quad \frac{\partial x_{1}^{\prime}}{\partial v}=-\frac{l}{s} \frac{\partial x_{1}}{\partial v}, \S
$$

we also have

$$
e_{1}^{\prime}=-\frac{h}{t} e_{1}, \quad g_{1}^{\prime}=-\frac{l}{s} g_{1} .
$$

Hence, since $C=t / s$,

$$
H_{2}=\frac{e_{1} g}{g_{1} e} \cdot \frac{1}{C}
$$

* Eisenhart, T. S., $\$ 837,38$ and $\$ \S 51,52$.

$\dagger$ Eisenhart, T. S., \& 15.

¥Cf. W. C. Graustein, $A n$ invariant of a general transformation of surfaces, \& 5.

\& Eisenhart, T. S., \& 15 . 
As a result

$$
H=H_{1} H_{2} H_{3} .
$$

THEOREM V. The product of the invariants $B$ of the parallel, radial, and a second parallel transformation into which a transformation $F$ can be factored (in Eisenhart's way) is equal to the invariant $B$ of the transformation $F$.

The transformation $F$ as considered by Jonas* was built up of a radial transformation $R_{1}$, a parallel transformation $P_{2}$, and another radial transformation $R_{3}$; i.e.,

$$
N \stackrel{R_{1}}{\rightarrow} \bar{N} \stackrel{P_{1}}{\rightarrow} \bar{N}^{\prime} \stackrel{R_{1}}{\rightarrow} N_{1} .
$$

In this case also Graustein $\dagger$ has shown that the invariant $C$ of the transformation $F$ is equal to the product of those of $R_{1}, P_{2}, R_{\mathbf{3}}$.

The invariant $B$ for $P_{2}$, i.e. $H_{2}$, is unity since $P_{2}$ is a perspective transformation. For $R_{1}$ and $R_{3}, C_{1}=C_{3}=1$. Hence, from (9.2),

$$
H_{1}=\frac{\bar{e} g}{\bar{g} e}, \quad H_{3}=\frac{e_{1} \bar{g}^{\prime}}{g_{1} \bar{e}^{\prime}} .
$$

But $\bar{e}^{\prime}=t \bar{\varepsilon}, \bar{g}^{\prime}=s \bar{g} . \neq$ Thus, again,

$$
H=H_{1} H_{2} H_{3} .
$$

THEOREM VI. The product of the invariants $B$ of the radial, parallel and second radial transformation into which a transformation $F$ can be factored (in Jonas' way) is equal to the invariant $H$ of the transformation $F$.

We are led to the conclusion from these facts that the methods of Eisenhart and Jonas are duals of one another.

\section{NETS OF SPECIAL TYPE}

15. Nets with equal invariants. The point equation of the net $N_{1}$ determined in $\$ 1$ as an $F$ transform of the net $N$ having (1.1) as its point equation is

$$
\frac{\partial^{2} \theta_{1}}{\partial u \partial v}=\frac{\partial}{\partial v} \log \left(\frac{a t}{\theta^{\prime}}\right) \frac{\partial \theta_{1}}{\partial u}+\frac{\partial}{\partial u} \log \left(\frac{b s}{\theta^{\prime}}\right) \frac{\partial \theta_{1}}{\partial v} \cdot \S
$$

* Cf. footnote on Jonas, Introduction.

$\dagger$ W. C. Graustein, $A n$ invariant of a general transformation of surfaces.

$\ddagger$ Cf. Eisenhart, T. S., \& 16, (21).

\& Eisenhart, T. S., \& 15 . 
If the point equation of $N(1.1)$ has equal point invariants, ${ }^{*}$

$$
\frac{\partial^{2}}{\partial u \partial v} \log \left(\frac{a}{b}\right)=0,
$$

and conversely. Thus $N_{1}$ will also have equal point invariants if and only if

$$
\frac{\partial^{2} \log C}{\partial u \partial v}=0 \text {; i.e. } C=\frac{U(u)}{V(v)},
$$

where $U$ is a function of $u$ alone and $V$ of $v$ alone.

The net $N$ whose tangential equation is (8.3) has equal tangential invariants $\dagger$ if and only if

$$
\frac{\partial^{2}}{\partial u \partial v} \log \left(\frac{\alpha}{\beta}\right)=0 .
$$

Hence $N_{1}$ having (11.6) as its tangential equation will also have equal tangential invariants if and only if

$$
\frac{\partial^{2} \log H}{\partial u \partial v}=0 \text {; i.e. } H=\frac{U(u)}{V(v)} .
$$

TheOREM VII. An $F$ transform of a net $N$ having equal point [tangential] invariants will also have equal point [tangential] invariants if and only if the invariant $C[H]$ of the transformation is of the form $U(u) / V(v)$.

16. Transformations $F$ with constant invariants. Consider two transformations $F$ of a net $N$ by means of the same solution $\theta$ of its point equation. Let the two invariants $C$ of these transformations, $C_{1}$ and $C_{2}$, be constant. Equation (3.2) yields

$$
\begin{aligned}
& \left(\frac{1}{C_{i}}-1\right) \frac{\partial^{2}}{\partial u \partial v} \log \left(\frac{\theta}{a}\right) \\
& +\left(1-C_{i}\right) \frac{\partial^{2}}{\partial u \partial v} \log \left(\frac{\theta}{b}\right)=0 \quad(i=1,2) .
\end{aligned}
$$

Thus, either

or

(ii)

$$
\frac{\partial^{2}}{\partial u \partial v} \log \left(\frac{\theta}{a}\right)=0 \quad \text { and } \quad \frac{\partial^{2}}{\partial u \partial v} \log \left(\frac{\theta}{b}\right)=0 ;
$$

* Eisenhart, T. S., $\$ 6$.

† Eisenhart, T. S., \& 53.

$$
\left|\begin{array}{ll}
\frac{1}{C_{1}}-1 & 1-C_{1} \\
\frac{1}{C_{2}}-1 & 1-C_{2}
\end{array}\right|=0 .
$$


If (i) obtains, $N$ and the two $F$ transforms have equal point invariants. If, then, $N$ has unequal point invariants, (ii) must hold; i.e.,

$$
\left(1-C_{1}\right)\left(1-C_{2}\right)\left(C_{1}-C_{2}\right)=0 .
$$

From this result and its dual we are led to

THEOREM VIII. If, in a harmonic[conjugate] triad of nets with unequal point [tangential] invariants, the invariants $C[B]$ of the three transformations $F$ are constant, at least one of the transformations is radial [perspective].

However, if (ii) does not hold, (i) must, and $N$ will have equal point invariants. The $F$ transforms will also have equal point invariants (cf. \$15).

TheORem IX. If a net $N$ admits of two non-radial [non-perspective] transformations $F$ by means of a given solution $\theta[\lambda]$ of its point [tangential] equation with constant but unequal invariants $C[H]$, the net $N$ has equal point [tangential] invariants; and the $F$ transforms also have equal point [tangential] invariants.

17. Transformations $K$ and $\Omega$. A transformation $F$ for which $C=-1$ is called a transformation $K,{ }^{*}$ and one for which $B=-1$, a transformation $\Omega . \dagger$ For a transformation $K$, equation (3.2) yields

$$
\frac{\partial^{2}}{\partial u \partial v} \log \left(\frac{a}{b}\right)=0 ;
$$

and for a transformation $\Omega$, equation (11.1) becomes

$$
\frac{\partial^{2}}{\partial u \partial v} \log \left(\frac{\alpha}{\beta}\right)=0 .
$$

Thus we have the known fact that two nets in relation $K[\Omega]$ have equal point [tangential] invariants.

If $N$ has equal point invariants its point equation is of the form

$$
\frac{\partial^{2} \theta}{\partial u \partial v}=\frac{\partial \log \psi}{\partial v} \frac{\partial \theta}{\partial u}+\frac{\partial \log \psi}{\partial u} \frac{\partial \theta}{\partial v} .
$$

Equation (3.2) for a constant invariant $C$ of an $F$ transformation of such a net yields

* Eisenhart, T. S., $\$ 25$.

$\dagger$ Eisenhart, T. S., $\$ 53$. 


$$
\left(\frac{1}{C}-C\right) \frac{\partial^{2}}{\partial u \partial v} \log \left(\frac{\theta}{\psi}\right)=0 .
$$

We may say that in general

$$
\frac{\partial^{2}}{\partial u \partial v} \log \left(\frac{\theta}{\psi}\right) \neq 0 .
$$

This fact and its dual leads to

TheOREM X. A non-radial[non-perspective] transformation $F$ of a net with equal point [tangential] invariants having a constant invariant $C[B]$ is, in general, a transformation $K[\Omega]$.

18. The product of the invariants $C$ and $H$. From (9.2) we see that if $C H=1$, we have

$$
\frac{e_{1}}{g_{1}}=\frac{e}{g}
$$

Hence

THEOREM XI. If two nets correspond by a transformation $F$ for which the product of the invariants $C$ and $B$ is unity, the surfaces of the nets are so mapped that their asymptotic lines correspond. Conversely, if the surfaces of two nets in relation $F$ are so mapped that their asymptotic lines correspond, the product of the invariants $C$ and $H$ of the transformation $F$ is unity.

From (9.2) we conclude

THEOREM XII. If the parameters of one of two nets in relation $F$ are isothermal-conjugate, ${ }^{*}$ those of the other net are also isothermal-conjugate if and only if the product of the invariants $C$ and $H$ of the transformation $F$ is unity.

We can go a step farther. A net is isothermal-conjugate if, when parametric, $e / g=U(u) / V(v) . \dagger$ Consequently, using Theorem XII and (9.2) we have

THEOREM XIII. If the nets $N$ and $N_{1}$ in relation $F$ have three of the following four properties, they have the fourth also:

(a) $N$ and $N_{1}$ have equal point invariants;

(b) $N$ and $N_{1}$ have equal tangential invariants;

(c) $N$ is isothermal-conjugate;

(d) $N_{1}$ is isothermal-conjugate.

* Eisenhart, Differential Geometry, pp. 198-199.

$\dagger$ Eisenhart, Differential Geometry, loc. cit. 
If (a) [(b)] holds and (c) and (d) both hold, and one of the nets $N$ and $N_{1}$ has equal tangential[point] invariants, then the other net also has equal tangential [point] invariants.

We may write (9.2) in the form

$$
\begin{aligned}
C H & =\frac{e_{1} g_{1}}{D_{1}^{2}} \cdot \frac{D^{2}}{e g} \cdot \frac{g^{2} D_{1}^{2}}{g_{1}^{2} D^{2}} \\
& =\frac{K_{1}}{K} \cdot\left(\frac{g D_{1}}{g_{1} D}\right)^{2},
\end{aligned}
$$

where $K$ and $K_{1}$ are the Gaussian curvatures of the surfaces carrying $N$ and $N_{1}$.

ThEOREM XIV. If the nets $N$ and $N_{1}$ in relation $F$ are real and the parameters are real, their surfaces have their Gaussian curvatures of the same or opposite sign according as the product $\mathrm{CH}$ of the transformation is positive or negative.

\section{TRANSFormations of RibadCoUR}

19. $O$-nets and conjugate normal congruences. The curves of a net $N$ form an orthogonal system; i.e., $N$ is an $O$-net, if and only if they are the lines of curvature of the surface of $N$. The point equation of the $O$-net of a surface is

$$
\frac{\partial^{2} \theta}{\partial u \partial v}=\frac{\partial \log E^{1 / 2}}{\partial v} \frac{\partial \theta}{\partial u}+\frac{\partial \log G^{1 / 2}}{\partial u} \frac{\partial \theta}{\partial v},
$$

and its tangential equation is

$$
\frac{\partial^{2} \lambda}{\partial u \partial v}=\frac{\partial}{\partial v} \log \frac{e}{E^{1 / 2}} \frac{\partial \lambda}{\partial u}+\frac{\partial}{\partial u} \log \frac{g}{G^{1 / 2}} \frac{\partial \lambda}{\partial v} .
$$

The congruence of normals to a surface is conjugate to its $O$-net. In fact the spherical representation of this $O$-net $N^{*}$ serves as a parallel net $N^{\prime}$ whose coördinates are direction parameters of this normal conjugate congruence. Let $\zeta$ be the direction cosines of the normals:

$$
\frac{\partial \zeta}{\partial u}=-\frac{e}{E} \frac{\partial x}{\partial u}, \quad \frac{\partial \zeta}{\partial v}=-\frac{g}{G} \frac{\partial x}{\partial v} .
$$

\footnotetext{
"The surface of $N$ is assumed to be neither a sphere nor a developable.
} 
If $\theta$ is an arbitrary solution of (19.1), equations

$$
\frac{\partial p}{\partial u}=-\frac{e}{E} \frac{\partial \theta}{\partial u}, \quad \frac{\partial p}{\partial v}=-\frac{g}{G} \frac{\partial \theta}{\partial v}
$$

are compatible, and a function $p$ so defined is a solution of the point equation of tue spherical representation $N^{\prime}$ of the $O$-net $N$. Hence an arbitrary $F$ transform $N_{0}$ of $N$ along its normal conjugate congruence has the coördinates

$$
x_{0}=x-\frac{\theta}{p} \zeta \text {. }
$$

For the surface of $N_{0}$,

$$
F_{0}=\frac{\partial \theta_{0}}{\partial u} \frac{\partial \theta_{0}}{\partial v}
$$

where

$$
\theta_{0}=-\frac{\theta}{p}
$$

If $\partial \theta_{0} / \partial u=\partial \theta_{0} / \partial v=0, \theta_{0}$ is constant, and the net $N_{0}$ of (19.5) is parallel to $N$.

If $\partial \theta_{0} / \partial u=0, \partial \theta_{0} / \partial v \neq 0$, equations (19.7) and (19.4) show that $\partial \theta / \partial u=0$, $\partial \theta / \partial v \neq 0$. Thus $\theta_{0}$ and $\theta$ are functions of $v$ alone. In this case we have for the directions of the curves of $N_{0}^{*}$

$$
\begin{aligned}
& \frac{\partial x_{0}}{\partial u}=-\left(\frac{e \theta_{0}}{E}-1\right) \frac{\partial x}{\partial u}, \\
& \frac{\partial x_{0}}{\partial v}=\left(\frac{g \theta_{0}-G}{G p}\right)\left(\zeta \frac{\partial \theta}{\partial v}-p \frac{\partial x}{\partial v}\right) .
\end{aligned}
$$

Such a transformation is of a type studied by Graustein $†$ and termed by him a semi-parallel map.

THEOREM XV. A necessary and sufficient condition that an $F$ transform of an $O$-net along its normal conjugate congruence be an $O$-net is that the transformation be either parallel or semi-parallel.

* Eisenhart, T. S., \& 15, (19).

† W. C. Graustein, Semi-parallel maps of surfaces, Annals of Mathematics, (2), vol. 27 (1926), p. 271. 
20. Transformations $R$. If $N^{\prime}:\left(x^{\prime}\right)$ is an arbitrary net parallel to the $O$-net $N$, the equations

$$
\frac{\partial \theta}{\partial u}=\left(x^{\prime} \mid \frac{\partial x}{\partial u}\right), \quad \frac{\partial \theta}{\partial v}=\left(x^{\prime} \mid \frac{\partial x}{\partial v}\right)
$$

are compatible, and $\theta$ will satisfy (19.1). The function

$$
\theta^{\prime}=\frac{\left(x^{\prime} \mid x^{\prime}\right)}{2}
$$

is a "corresponding" solution of $N^{\prime}$ in the sense of $\$ 1$. Thus we may obtain a net $N_{1}:\left(x_{1}\right)$

$$
x_{1}=x-\frac{2 \theta}{\left(x^{\prime} \mid x^{\prime}\right)} x^{\prime}
$$

as an $F$ transform of $N$. Here $N_{1}$ is also an $O$-net.

As a matter of fact, the surfaces of the $O$-nets $N$ and $N_{1}$ are the sheets of the envelope of a two-parameter system of spheres, the curves of $N$ and $N_{1}$ being the loci of the points of contact of the spheres.* This transformation $F$ of $N$ into $N_{1}$, as indicated by (20.3), is termed a transformation of Ribaucour, or briefly, a transformation $R$.

The invariants $C$ and $H$ of a transformation $R$ enter in the symmetrical relations

$$
C^{2}=\frac{E_{1} G}{G_{1} E}, \quad C H=\frac{e_{1} g}{g_{1} e}, \quad H^{2}=\frac{\varepsilon_{1} G}{G_{1} \varepsilon} \cdot \dagger
$$

The points with coordinates $\zeta$ and $\zeta_{1}$, the direction cosines of the normals to $N$ and $N_{1}$, trace $O$-nets on the unit sphere which are in relation $F$. $f$ The point equations of these nets on the unit sphere are the same as their tangential equations and are also equal to the tangential equations of the nets $N$ and $N_{1}$. 'Thus

TheOREM XVI. The invariant $H$ of a transformation $R$ is equal to the invariant $C(=H)$ of the transformation $F$ existing between the spherical representations of the nets in the relation $R$.

* Eisenhart, T. S., $\$ \$ 68-72$.

$+\mathcal{E}, \mathcal{F}, G$ are the coefficients of the square of the linear element of the spherical representation of the surface of $S$.

$\ddagger$ Eisenhart, T. S., \& 78, (11). 
21. Applications. We return to the equations (20.4), and exclude radial and perspective transformations.

If the transformation $R$ is also $K, C=-1$ and

$$
\frac{E_{1}}{E}=\frac{G_{1}}{G}
$$

and conversely. Since $N$ and $N_{1}$ have equal point invariants (cf. $\$ 17$ ), their surfaces are isothermic. Conversely, if the surfaces of $O$-nets in relation $R$ are isothermic, we can choose parameters so that (21.1) holds. Moreover, (21.1) is the condition that the map be conformal.

The following theorems are thus readily obtained:

TheOREM XVII. A necessary and sufficient condition that the surfaces of $O$-nets in relation $R$ be conformally mapped is that both surfaces be isothermic. The transformation is then also $K$. $^{*}$

TheOREm XVIII. A necessary condition that the two surfaces whose O-nets are in relation $R$ be isometrically mapped is that the transformation be also $K$. Both surfaces are then isothermic.

Theorem XIX. A necessary and sufficient condition that the spherical representations of two surfaces whose O-nets are in relation $R$ be conformally mapped is that the transformation be also $\Omega$.

TheOREM XX. A necessary condition that the spherical representations of two surfaces whose $O$-nets are in relation $R$ be isometrically mapped is that the transformation $R$ be also $\Omega$.

Finally we have

TheOREM XXI. If two surfaces whose $O$-nets are in relation $R$ are mapped conformally (or isometrically) and if their spherical representations are also so mapped, the transformation $R$ is both $K$ and $\Omega$; and the surfaces and their spherical representations are isothermic. Conversely, if these surfaces and their spherical representations are isothermic, the surfaces are conformally mapped and so also are their spherical representations, and the transformation $R$ is both $K$ and $\Omega$. In these cases of a transformation $R$ being both $K$ and $\Omega$, the asymptotic lines of both surfaces correspond. $\dagger$

* Theorem of Cosserat, Eisenhart, T. S. $\$ 82$ and footnote (61).

$\dagger$ Cf. Theorem XI. 
If the $O$-net of a minimal surface is parametric,

$$
\frac{E}{G}=-\frac{e}{8} .
$$

Thus we are led to

THEOREM XXII. When the $O$-nets of two minimal surfaces are in relation $R$, the invariants $C$ and $H$ of the transformation are equal; and, conversely, if the invariants $C$ and $B$ of $a$ transformation $R$ are equal and the surface of one of the O-nets is minimal, so is the surface of the second.

Harvard University,

Cakbrigge, Mass. 